\title{
Periodic Epidemic Spreading over Complex Systems: Modeling and Analysis
}

\author{
Zhe Wang, ${ }^{1}$ Hong Yao, ${ }^{2}$ Haiyan Han, ${ }^{1}$ Jun Du, ${ }^{1}$ and Chao Ding \\ ${ }^{1}$ Aeronautics and Astronautics Engineering College, AFEU, Xi'an 710038, China \\ ${ }^{2}$ Science College, AFEU, Xian 710051, China \\ Correspondence should be addressed to Zhe Wang; thisiswangzhe@qq.com
}

Received 23 February 2016; Revised 6 June 2016; Accepted 26 July 2016

Academic Editor: Nicolas J. Leconte

Copyright (c) 2016 Zhe Wang et al. This is an open access article distributed under the Creative Commons Attribution License, which permits unrestricted use, distribution, and reproduction in any medium, provided the original work is properly cited.

\begin{abstract}
It is well observed that some infectious diseases show a feature of periodicity; that is, the disease may prevail during a certain season and vanish afterwards. In the effort of understanding this specific phenomenon, in this paper, we propose a spreading model with a time-variant infectivity function considering both the features of periodicity and agent variability. We apply the modified model to a scale-free network frame with tunable power-law coefficient to find out the characteristics of periodic spreading dynamics. Our work consists of both theoretical derivation and a series of corresponding numerical simulations in order to find out the influence of the parameters of both network topology and the modified model on spreading dynamics in the underlined networks. The experiments prove the success of our model in producing a periodic behavior of spreading, and the results agree well with theoretical calculations.
\end{abstract}

\section{Introduction}

The spreading process on top of complex systems is one of the most focused subjects in the realm of network science and intensively studied [1-6]. A large range of realistic systems such as communication network, transportation web, power grid, or large-scale supply chains [7-9] could undergo some sort of spreading processes and cause potential economic losses. The last fifteen years have seen the successful application of complex network theory on the analysis of dynamical behaviors on top of complex systems [10-14]. From the perspective of this theory, the individuals of a real-world complex system are modeled by agents in a network and the contacts among them are considered as the links connecting agents. A healthy agent may become infectious through the already infected agents that are linked to it. On this common basis, researchers have integrated the network view into a series of compartmental models like SI (SusceptibleInfected), SIS (Susceptible-Infected-Susceptible), and SIR (Susceptible-Infected-Recovered) among many others and have unveiled some exciting features of networked spreading dynamics [15-17]. Reference [18] considered the effect of medium by means of a modified SIS model and recovered that spreading medium could accelerate epidemic spreading without changing the final spreading scale. Reference [19] established R-SIR-R (Risk-SIR-Random) and R-SIR-A (Risk-SIRAcquaintance) on the basis of cellular automata to study the safety risk spreading of complex information system on top of nearest coupled network, random network, WS small-world network, and BA scale-free network and concluded that the outbreak threshold decreases with the strengthening of network's heterogeneity. Reference [4] built a SVIR (SusceptibleVaccinated-Infected-Recovered) model and compared various vaccination strategies; the results showed that both the vaccination level and infection level are lower when adopting continuous strategy other than pure strategy.

Nevertheless, the compartmental models are too simplified to describe various spreading processes in real-world systems partly because the infectivity is treated as linear. To this end, nonlinear infectivity is proposed and many delicate models are put forward. Chu et al. in [20] proposed a epidemic spreading model in weighted SF network; they investigated the nonlinear infectivity and weighted transmission rate and found the strong effect of the infectivity parameter 
on the epidemic threshold and the prevalence. Reference [21] investigated a new SIS model with nonlinear infectivity as well as birth and death of agents and links, and the reproductive number is calculated mathematically.

In spite of the intensive research, many valuable problems still remain open. Why do some popular diseases (such as flu) show a notable feature of periodicity? What is the mechanism of this phenomenon? Note that a large number of spreading processes in practical systems display a feature of periodicity $[22,23]$. Reference [22] studied the spreading in community networks, and Zhou et al. found periodic behaviors in both SIS and SIRS models when the infection rate is larger than the threshold which may be helpful in understanding the spatial-temporal pattern of DHF incidence in Thailand. Reference [23] proposed a modified SIRS (Susceptible-InfectedRecovered-Susceptible) model and found that the timedelay of the assessment of epidemic risk can cause periodic outbreak of diseases. In the above papers, researchers tried to demonstrate the mechanism of periodicity from their own perspectives; however, they all adopted a constant effective infectivity rate in the spreading model regardless of the fact that the contagion rate of epidemics could be time-variant. Moreover, agent variability is also advised to be considered in the confirmation of effective infectivity rate.

In this paper, we take both the factors into account. We apply the new model to topologically alterable scale-free networks and discuss the influence of network's structural parameters, agent variability, and periodicity on the epidemic spreading behaviors. The results show that our model is successful in describing the periodic feature, and some influential parameters are proposed and investigated.

\section{Modified SIS Model}

There are two sorts of agents in SIS model (S and I) which are susceptible and infected. In this case, a susceptible agent can be infected when it is in contact with an already infected one with probability $\eta$. Once infected, agents recover with some probability $\beta$ per unit time. The dynamics of SIS model can be described by the following system of ordinary differential equations:

$$
\begin{aligned}
& \frac{\mathrm{d} s(t)}{\mathrm{d} t}=-\eta i(t) s(t)+\beta i(t), \\
& \frac{\mathrm{d} i(t)}{\mathrm{d} t}=\eta i(t) s(t)-\beta i(t),
\end{aligned}
$$

where $s(t) \quad(i(t))$ indicates the number of susceptible (infected) agents at moment $t$. Without loss of generality, let $\beta=1$; hence the effective infectivity rate $\lambda=\eta / \beta=\eta$. Based on mean field theory, we can get the main equation:

$$
\frac{\mathrm{d} \rho_{k}(t)}{\mathrm{d} t}=-\rho_{k}(t)+\lambda k\left[1-\rho_{k}(t)\right] \Theta(\rho(t)),
$$

where the first term on the right side is the extinction term and the second one is the production term. The meaning of each parameter is as follows: (a) $\rho_{k}(t)$ is the probability that the agent of degree $k$ gets affected; (b) $\lambda$ is the probability that a healthy agent becomes infected when being in contact with an infected agent; (c) $\rho(t)=\sum_{k} P(k) \rho_{k}(t)$ is the average proportion of all the infected agents in the network and can be used to measure the spreading prevalence. Here, we call it spreading scale in the remaining part of this paper and take it as an important meter of spreading dynamics; (d) $\Theta(\rho(t))$ is the probability that a given edge of a certain agent is pointed to an infected neighbor. have

When the degree-degree interrelationship is weak, we

$$
\Theta=\sum_{k} \frac{k P(k) \rho_{k}(t)}{\langle k\rangle},
$$

where $\langle k\rangle$ is the mean degree of the network. Apparently, $\Theta \in[0,1]$; the sufficient condition that the spreading process sustains in the system is $\mathrm{d} \rho_{k}(t) / \mathrm{d} t \geq 0$. We take the critical value $\mathrm{d} \rho_{k}(t) / \mathrm{d} t=0$; then we have

$$
\rho_{k}=\frac{\lambda k \Theta}{1+\lambda k \Theta} .
$$

Taking agent variability into consideration, it requires

$$
\lambda(k)=\frac{\langle\lambda(k)\rangle k^{\alpha}}{\left\langle k^{\alpha}\right\rangle},
$$

where $\alpha<0$, and $\langle\lambda(k)\rangle$ is the average of $\lambda(k)$. We name $\alpha$ the agent variability weight for it defines the extent of agent difference. To make sure that $\lambda \in(0,1)$, we need two conditions to be satisfied:

$$
\begin{aligned}
& \text { Condition 1: }\langle\lambda\rangle<(\gamma-1) /(\gamma-\alpha-1) . \\
& \text { Condition 2: } k_{\min }>(\gamma-\alpha-3) /(\gamma-2),
\end{aligned}
$$

where $k_{\min }$ is the smallest degree of all agents and $\gamma$ the power-law coefficient. The above inequations are easy to be satisfied in practice.

After agent variability, we take periodicity into consideration by modifying (5) as

$$
\lambda(k, t)=\frac{\langle\lambda(k, t)\rangle k^{\alpha}}{\left\langle k^{\alpha}\right\rangle}[1+\varepsilon f(t)] .
$$

$f(t)$ is a continuous function with period $T,-1 \leq f(t) \leq$ 1 ; $\varepsilon$ is the amplitude, $0 \leq \varepsilon \leq 1$. Also, we have $\int_{a}^{a+T} f(t) \mathrm{d} t=$ $0, \forall a \in R$.

Revising (4), we can get

$$
\rho_{k}=\frac{\lambda(k, t) k \Theta}{1+\lambda(k, t) k \Theta} .
$$

Integrating (7) into (3), we will have an autonomous equation of $\Theta$ :

$$
\Theta=\sum_{k} \frac{P(k)}{\langle k\rangle} \cdot \frac{\lambda(k, t) k^{2} \Theta}{1+\lambda(k, t) k^{2} \Theta} .
$$

There exists a trivial solution $\Theta=0$. To allow for nontrivial solutions, there must be

$$
\frac{\mathrm{d}}{\mathrm{d} \Theta}\left(\sum_{k} \frac{P(k)}{\langle k\rangle} \cdot \frac{\lambda(k, t) k^{2} \Theta}{1+\lambda(k, t) k^{2} \Theta}\right)_{\Theta=0} \geq 1 .
$$


Clearly, there is

$$
\frac{\left\langle\left(\langle\lambda\rangle k^{\alpha} /\left\langle k^{\alpha}\right\rangle\right)[1+\varepsilon f(t)] k^{2}\right\rangle}{\langle k\rangle} \geq 1 .
$$

With $\left\langle\left(\langle\lambda\rangle k^{\alpha} /\left\langle k^{\alpha}\right\rangle\right)[1+\varepsilon f(t)] k^{2}\right\rangle /\langle k\rangle \geq\left\langle\left(\langle\lambda\rangle k^{\alpha} /\right.\right.$ $\left.\left.\left\langle k^{\alpha}\right\rangle\right)(1-\varepsilon) k^{2}\right\rangle /\langle k\rangle$, we obtain the sufficient condition of $(10)$ :

$$
\frac{\left\langle\left(\langle\lambda\rangle k^{\alpha} /\left\langle k^{\alpha}\right\rangle\right)(1-\varepsilon) k^{2}\right\rangle}{\langle k\rangle} \geq 1 .
$$

After solving it, we obtain

$$
\langle\lambda\rangle \geq \frac{\left\langle k^{\alpha}\right\rangle\langle k\rangle}{(1-\varepsilon)\left\langle k^{\alpha+2}\right\rangle} .
$$

The outbreak threshold that sustains the spreading process on top of the network comes as

$$
\lambda_{0 c}=\frac{\left\langle k^{\alpha}\right\rangle\langle k\rangle}{(1-\varepsilon)\left\langle k^{\alpha+2}\right\rangle} .
$$

$\lambda_{0 c}$ is related with $\alpha, \varepsilon$, and $k$. Comparing it with the threshold of traditional SIS model on top of scale-free network which is $\lambda_{c}=\langle k\rangle /\left\langle k^{2}\right\rangle$, it is quite clear that $\lambda_{0 c}>\lambda_{c}$. In other words, epidemics are more difficult to sustain on the network when adopting our modified model. Specially, $\varepsilon$ must to be set to a small value to avoid error enlargement.

\section{Experiments and Discussion}

In the above sections, we proposed the modified SIS spreading model which involves an abstract periodic function $f(t)$ $(-1 \leq f(t) \leq 1)$. Without loss of generality, let $f(t)=$ $\sin (\sigma \pi t)$ for simulative convenience's sake. We conduct the simulations by distributing initiative infected agents randomly on the network with probability $20 \%$.

3.1. Network Model. There was a prevailing consumption for a long period of time that all complex systems could be considered mathematically as random networks until Barabási and Albert [11] falsified that consumption. Their work on a WWW project indicates that the degree distribution of many practical networks submits power-law distribution and they proposed the BA scale-free network model with power-law coefficient fixed to 3 by preference attachment and growth mechanism. Although BA model is able to picture some realistic networks such as the Internet, most real-world complex systems which can be abstracted as scale-free networks do not submit a fixed coefficient power-law distribution. As a result, [24, 25] built a network construction mechanism which allows for a controllable power-law coefficient. The details are as follows.

By distributing a normalized weight to each of the $N$ isolated agents and adding links among the agents with some probability, we get a tunable power-law coefficient and mean degree by adjusting the total edge number. The algorithm is as follows.
Step 1. Distribute weight $q_{i}=i^{-\omega}$ to agent $i, i=1,2, \ldots, N$, where $N$ is the total number of agents; the tunable parameter $\omega$ is within interval $0 \leq \omega<1$. Normalize the weight as

$$
q_{i}^{*}=\frac{q_{i}}{\sum_{k} q_{k}} .
$$

Step 2. Add edges among agents. If agent $i$ and $j$ satisfy

$$
\begin{aligned}
& q_{i}^{*} \geq r_{1}>q_{i+1}^{*}, \\
& q_{j}^{*} \geq r_{2}>q_{j+1}^{*},
\end{aligned}
$$

where $r_{1}$ and $r_{2}$ are random numbers in $[0,1]$, and if $i$ and $j$ are not connected, add an edge between them. By this rule, isolated nodes with high weights are more likely to be linked.

Step 3. Repeat Step 2 till there are $m N$ edges.

The mean degree of this network is $2 m$, and the degree distribution satisfies power-law $P(k) \propto k^{-\gamma}$, where $k$ is the degree of agent and $\gamma$ satisfies

$$
\gamma=1+\frac{1}{\omega} .
$$

Apparently, we are able to adjust the network topology by tuning parameters $m$ and $\omega$.

3.2. Time Evolution. To reveal the new characteristics of the modified spreading model built in Section 2, we compare it with three comparative models in the following part and analyze their simulative performance. The models we are going to discuss are listed as follows:

Model A: SIS model with no periodic infectivity and no agent variability.

Model B: SIS model with periodic infectivity and no agent variability.

Model C: SIS model with agent variability and no periodic infectivity.

Model D: SIS model with both periodicity and agent variability.

The interactive scale-free network has the size $N=500$ and mean degree $\langle k\rangle=10$. Parameters in comparative spreading models are set as follows: $\sigma=0.01, \varepsilon=0.2$, $\alpha=-0.5$, and $\langle\lambda\rangle=0.5$ for periodic infectivity models and $\lambda=0.5$ for constant infectivity models.

The three curves shown in Figure 1 correspond, respectively, to the time evolution of Model A, Model B, and Model B's infectivity rate $\lambda$. Similarly, Figure 2 shows the time evolution of Models $C$ and $D$ and $\lambda$. In particular, the vertical location of $\lambda$ is adjusted to get a better view of comparison.

As shown in Figure 1, the evolution curve of Model A oscillates substantially within interval $(0.44,0.51)$ on a minor cycle which is about 2 time steps. Meanwhile, when considering periodicity in Model $\mathrm{B}$, a dual-cycle effect is observed on the curve: on one hand, the curve oscillates on a short cycle as in Model A; on the other hand, Model B curve synchronizes 


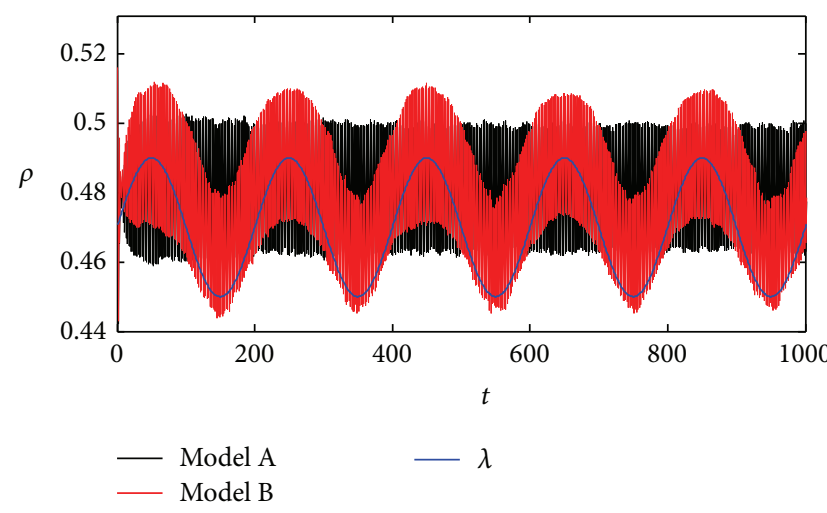

(a)

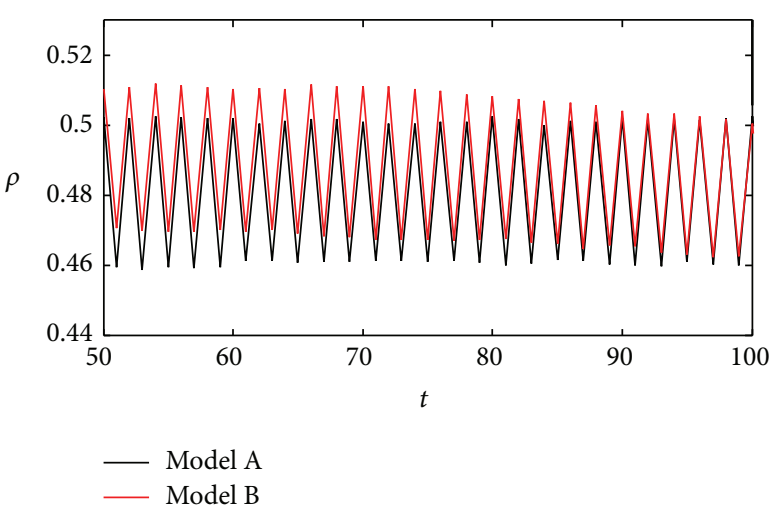

(b)

FIGURE 1: Evolution of Model A, Model B, and its infectivity rate $\lambda$. (a) For Model A, the spreading scale oscillates violently within the interval $(0.46,0.5)$ on a cycle of two time steps; nevertheless, Model B shows a more interesting feature; the evolution curve of Model B displays a dual-cycle characteristic where the spreading scale evolutes on a minor cycle and a major cycle simultaneously. (b) is a magnified view of the revolution of the two models from which we can clearly see the minor cycles.

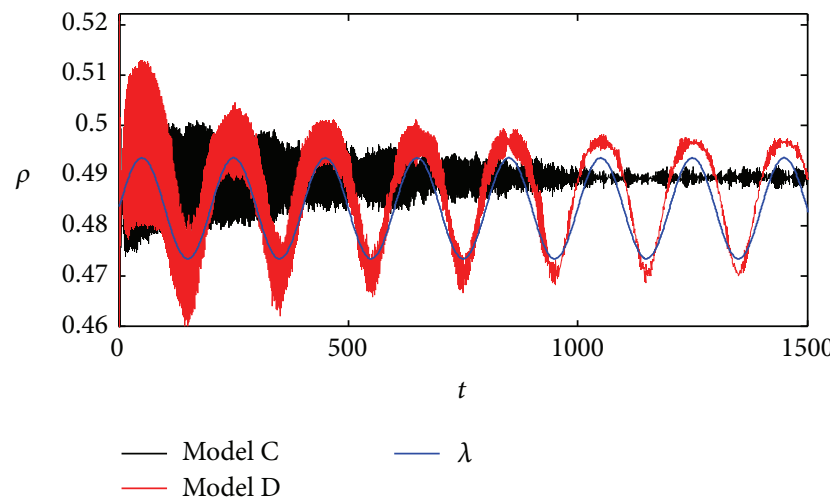

(a)

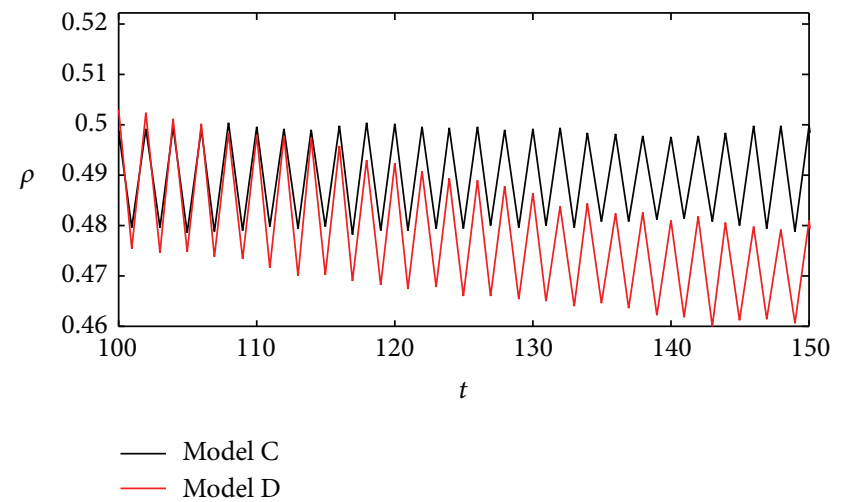

(b)

Figure 2: Evolution of Model C, Model D, and infectivity rate $\lambda$. In (a), it is shown that the evolution of both Models C and D is asymptotically stable where Model D curve is synchronized with the curve of infection rate. (b) is a magnified view of Models C and D.

with the infectivity rate on a major cycle which is 200 time steps according to our initial settings. By a simple modification of the SIS spreading model such as involving periodicity in infectivity, we can observe a realistic phenomenon in spreading dynamics that the spreading scale shows a periodic feature. In our modification, the infectivity rate $\lambda$ is a sine function instead of a constant number as in most researches, which causes the spreading scale to display a pseudo-sine function as well. Moreover, we have tested cosine function and several other functions for $\lambda$; we found the tendency of spreading scale during evolution in accordance with that of the infectivity rate $\lambda$ in all the results. To this end, we have reason to believe that our modified model on the infectivity rate provides an explanation for the periodic phenomenon in real-world spreading processes.

As to Models C and D, the simulation results are shown in Figure 2. After entering the steady stage, Model C curve is surprisingly steady and keeps in an almost horizontal state. Besides, Model D curve quite assembles that of Model A only with a smaller amplitude of the minor cycle. Moreover, if we take a close look at the curves in the figure, we can find that the amplitudes of both the models reduce as time goes on, but with a relatively low speed. For a better view, we compared Model A with $\mathrm{C}$ in Figure 3 where Model C involves agent variability and Model A does not and Model B with D in Figure 4 where Model D involves agent variability and Model $\mathrm{B}$ does not; by the clear difference shown in the figures, we can conclude with evidence that agent variability minishes the periodic amplitude.

3.3. The Influence of Network Topology. In the art of complex network, researchers' main focus lies in the interplay of network structure and the dynamics which occur on the network. In this subsection, we adopt the network model proposed in Section 2 and simulate the influence of powerlaw coefficient, mean degree, and so forth on the spreading dynamics. The initial setting of the modified SIS model is as follows: $\sigma=0.01, \varepsilon=0.2, \alpha=-0.5$. 


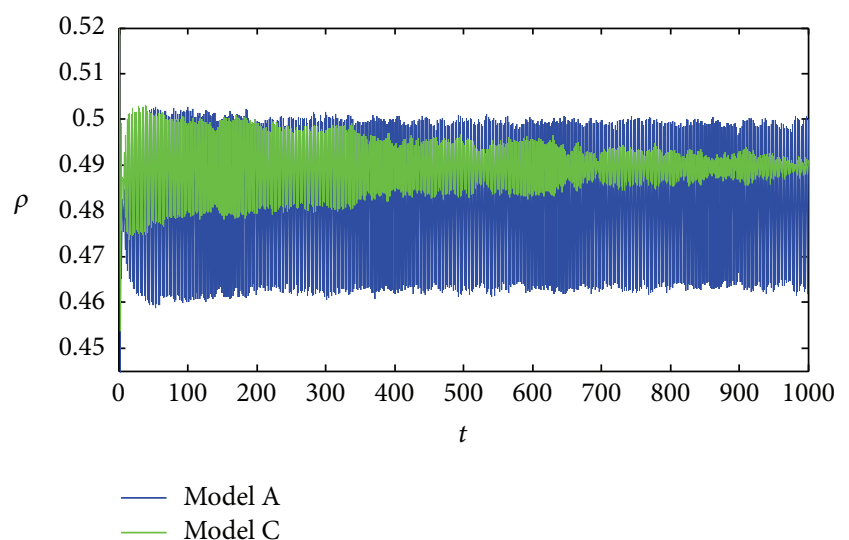

Figure 3: Comparison of Model A with Model C. In Model A, the spreading model considers no periodicity of infectivity or agent variability, while in Model $\mathrm{C}$ agent variability is taken into consideration.

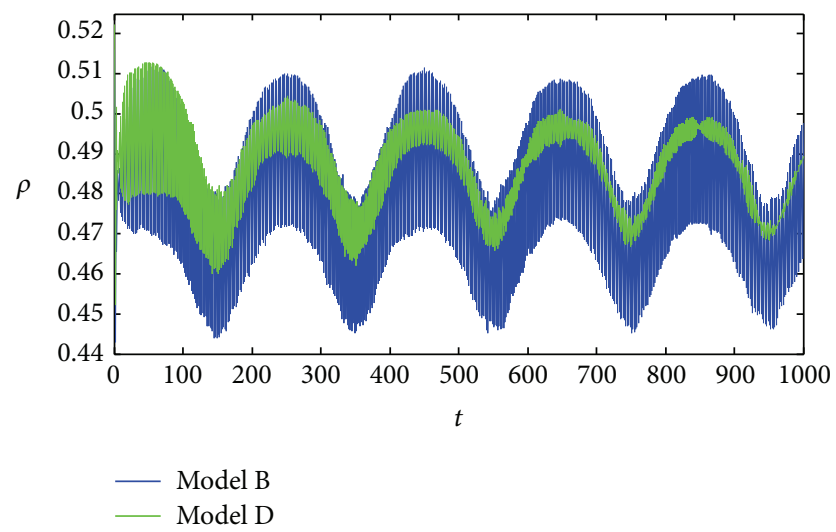

FIGURE 4: Comparison of Model B with Model D. In Model B, only infectivity periodicity is taken into consideration while Model D considers both infectivity periodicity and agent variability.

3.3.1. Power-Law Coefficient. The power-law coefficient is an indicator of homogeneity of a scale-free network. A big value of the coefficient corresponds to a high level of uniformity of degree distribution, and a more negative value of the coefficient corresponds to a low level of uniformity of degree distribution.

Figure 5 displays the plots of spreading scale $\rho$ with respect to $\langle\lambda\rangle$ under several values of $\gamma$. The simulation results indicate that they basically agree with the theoretical calculation by comparison of Figure 5 and (13), but the simulation thresholds are a bit larger than the theoretical ones; this is because the simulation curve is an average of 200 independent simulations; in some simulations the epidemic does not survive. Let us take the situation of $\gamma=2$ as an example, $\lambda_{0 c}=0.0609$ according to (13); meanwhile, in the figure, the black line starts to ascend in the interval [0.06, 0.07]. It is also observed from the same figure that $\rho$ is negatively related with $\gamma$, which means that the spreading scale descends with the homogeneity of the underlined network. This is because, in a more homogeneous network, the number of hub

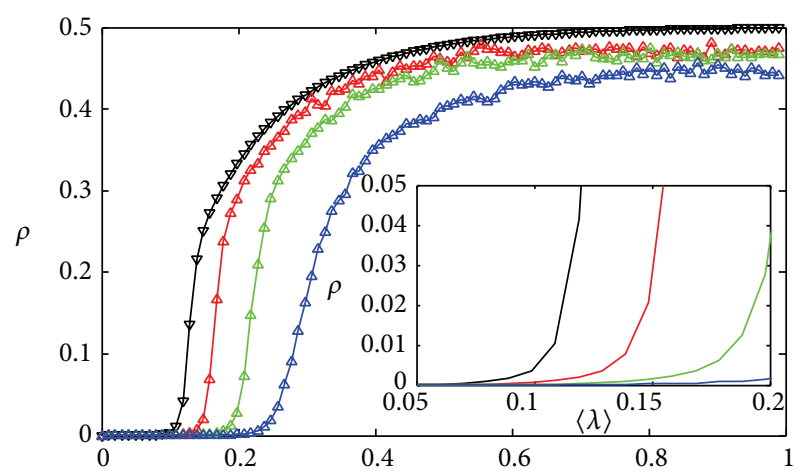

$\langle\lambda\rangle$

$$
\begin{array}{ll}
\rightarrow \gamma=2 & \triangle \gamma=4 \\
\triangle \gamma=3 & \triangle \gamma=5
\end{array}
$$

FIgURE 5: $\rho-\langle\lambda\rangle$ curve with variable $\gamma(t \rightarrow \infty)$. As the powerlaw coefficient increases, the spreading outbreaks more difficultly, indicating the enhancement of network robustness against epidemic propagation.

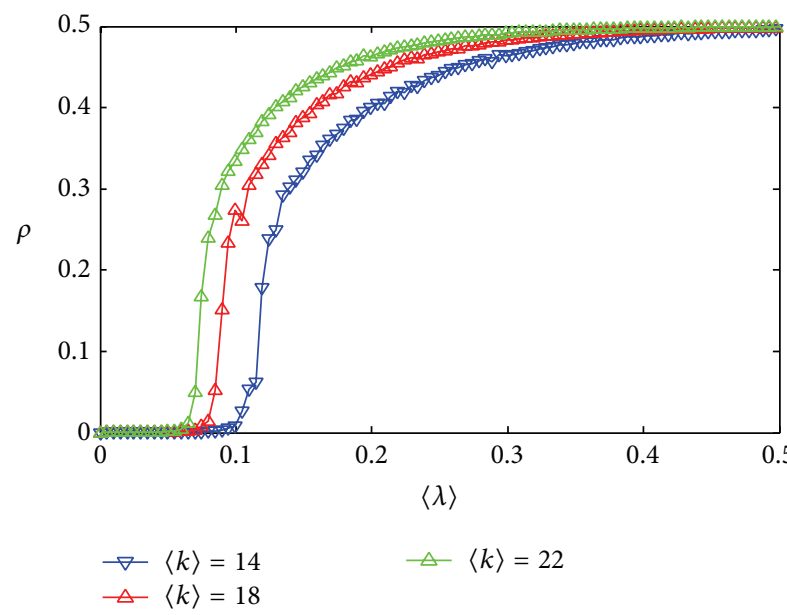

FIgURE 6: $\rho$ - $\langle\lambda\rangle$ curve with variable $\langle k\rangle(t \rightarrow \infty)$. The more innerconnected the network is, the more easily the epidemic breaks out.

nodes (those nodes with rather high degrees) is reduced; as a result, the influence of "super spreaders" on their neighbors is restrained which leads to a lower level of spreading.

3.3.2. Mean Degree of the Network. The mean degree indicates the average thickness of a network; this metric reflects the average connection number that a node has. The parameter setting of the spreading model is as follows: $\varepsilon=0.2$, $\sigma=0.01$, and $\alpha=-0.5$.

We can see that the influence of mean degree on the outbreak threshold is monotonous from Figure 6. The theoretical outbreak thresholds corresponding to $\langle k\rangle=14,\langle k\rangle=18$ and $\langle k\rangle=22$ are, respectively, $0.0797,0.0683$, and 0.0562 which are a bit smaller than simulation results due to the same averaging effect as in Section 3.3.1. The outbreak threshold is negatively related with mean degree of the network; in other words, the more connected the agents are to each other, 


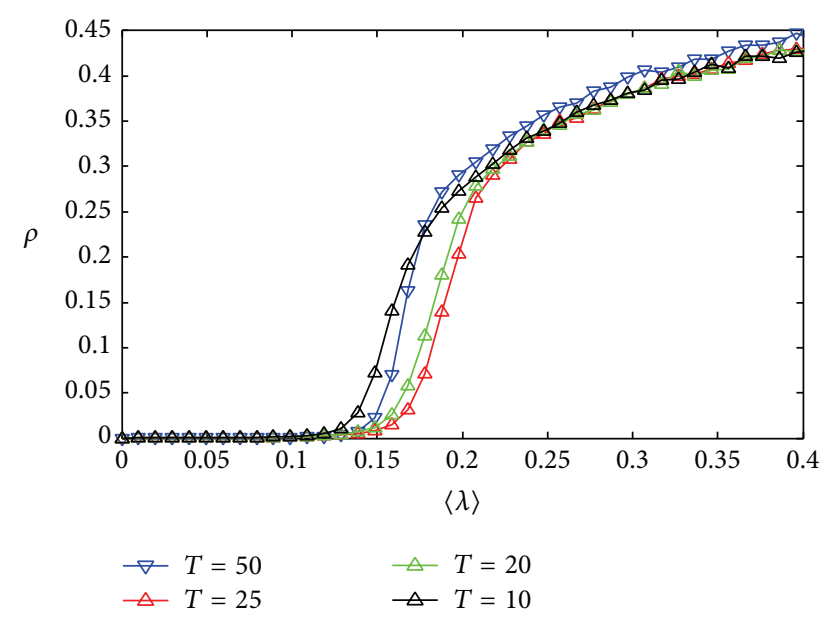

Figure 7: $\rho-\langle\lambda\rangle$ curve with variable $T(t \rightarrow \infty)$.

the easier the diseases break out and stay active in the system. The reason for this tendency is that a more highly connected network provides more routes for the spreading process to expand, which leads to a low outbreak threshold.

3.4. The Influence of Modified SIS Model. On the basis of the intensively studied linear infectivity, we propose a nonlinear periodic infectivity rate model with the consideration of agent variability and apply it to our network model. The modified spreading model we put forward includes two adjustable parameters: the cycle $T$ and agent variability weight $\alpha$.

3.4.1. Infectivity Rate Cycle. From the above discussion, we know the cycle of infectivity rate affects that of the spreading scale. In this subsection, we discuss its influence on the outbreak threshold and the spreading scale through numerical simulation. The parameter settings of the network and modified SIS model are as follows: $N=500,\langle k\rangle=10, \varepsilon=0.2$, and $\alpha=-0.5$.

The curves of $\rho$ with respect to $\langle\lambda\rangle$ when $T=50, T=20$, and $T=10$ are displayed in Figure 7. Clearly, the cycle of infectivity rate has little effect on the outbreak threshold and the final spreading scale. To illustrate this with more evidence, we simulated the time evolution of $\rho$ when $T=200$ and $T=50$ shown in Figure 8. It can be seen that after $t \geq 400$, the evolution enters a steady state. Both the curves are sinewave shaped and oscillate within the same interval; the only difference lies in the oscillation cycle.

3.4.2. Agent Variability Weight. In a realistic system, each agent's ability to avoid contagions varies because different agents have different local properties such as the degree property. Based on that idea, it is all natural to consider the infectivity rate as degree related. Obviously, it is reasonable to assume that agents with high degrees are more likely to contact with infected neighboring agents.

The interactive network we use has the minimum degree $k_{\min }=2$ which satisfies Condition 2 in Section 2. We plot $\rho$ versus $\langle\lambda\rangle$ when $\alpha=0, \alpha=-0.5, \alpha=-1$, and $\alpha=-1.5$.

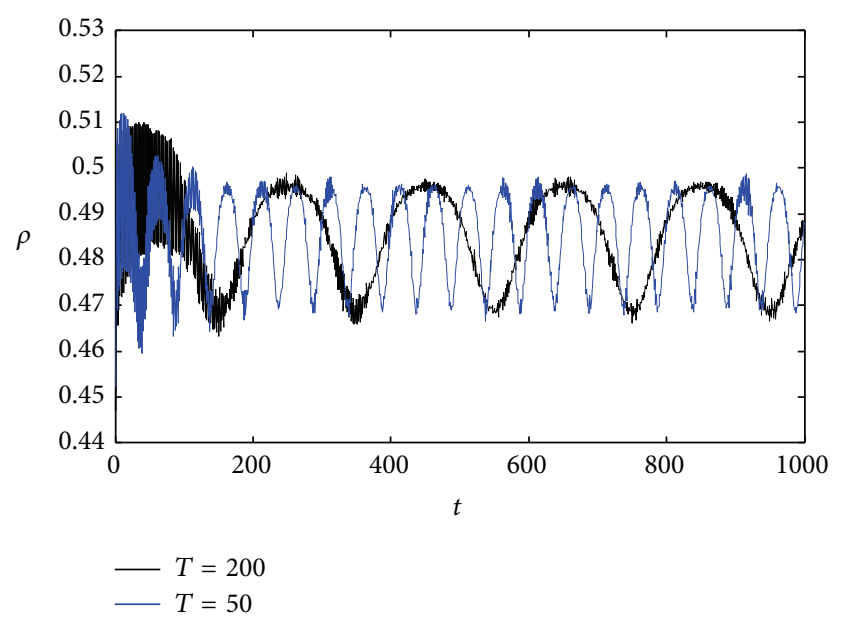

FIGURE 8: Evolution of $\rho$ with two kinds of periods. There is no difference in the oscillation amplitude between the cases when $T=$ 200 and when $T=50$.

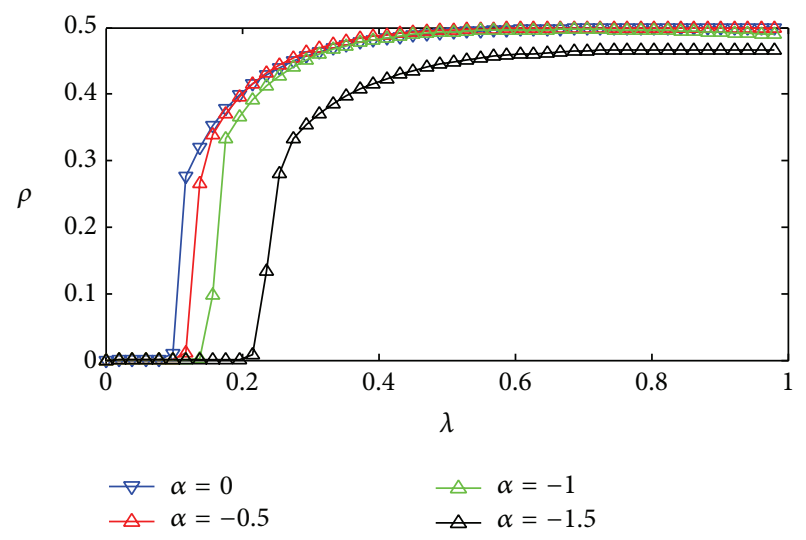

Figure 9: $\rho-\langle\lambda\rangle$ curve with variable $\alpha(t \rightarrow \infty)$.

The results are demonstrated in Figure 9. We compare the simulated thresholds to theoretical results which are 0.0677 , $0.0887,0.1152$, and 0.1511 and find that they fit well. As shown in (5), as $\alpha$ goes further from 0 , high degree agents are less likely to infect neighbors in accordance with (5) which causes the enhancement of network robustness against epidemic spreading outbreak.

\section{Conclusion}

The variation of infectivity rate of epidemic is a realistic consideration. Based on that, we modified the conventional model by adding periodic feature and agent variability to the infectivity function. We discussed the spreading dynamics of our model on top of topologically alterable scalefree networks by both theoretical and simulative measures. It is found that the periodic infectivity rate causes the final spreading scale to oscillate with the infectivity. Also, a dual-cycle phenomenon is observed; meanwhile, agent variability decreases the amplitude of the minor cycle. The network topology influences the spreading dynamics in 
several aspects. The power-law coefficient which indicates the heterogeneity of network has positive relationship with the spreading threshold but is positively related with the final spreading scale and the mean degree of network is negatively related with the threshold. Furthermore, the cycle of infectivity rate affects only the cycle of final spreading scale. Agent variability weight and infectivity periodic amplitude are both positively related with the spreading threshold.

\section{Competing Interests}

The authors declare that they have no competing interests.

\section{Authors' Contributions}

Z. Wang and H. Han wrote the paper and did the theoretical analysis, C. Ding did the simulation, and H. Yao and J. Du reviewed the paper.

\section{Acknowledgments}

This research is funded by the National Science Foundation of China (Grant no. 11447147).

\section{References}

[1] S. Chen, W. Huang, C. Cattani, and G. Altieri, "Traffic dynamics on complex networks: a survey," Mathematical Problems in Engineering, vol. 2012, Article ID 732698, 23 pages, 2012.

[2] M. Dickison, S. Havlin, and H. E. Stanley, "Epidemics on interconnected networks," Physical Review E, vol. 85, no. 6, Article ID 066109, 2012.

[3] M. Li, X. Du, A. E. Villaruz et al., "MRSA epidemic linked to a quickly spreading colonization and virulence determinant," Nature Medicine, vol. 18, no. 5, pp. 816-819, 2012.

[4] C.-R. Cai, Z.-X. Wu, and J.-Y. Guan, "Effect of vaccination strategies on the dynamic behavior of epidemic spreading and vaccine coverage," Chaos, Solitons \& Fractals, vol. 62-63, pp. 3643, 2014.

[5] Q. Guo, L. Li, Y. Chen, Y. Yang, and X. Niu, "Modeling dynamics of disaster spreading in community networks," Nonlinear Dynamics, vol. 64, no. 1-2, pp. 157-165, 2011.

[6] Z. Wang, H. Yao, G. Yang, and J. Du, "Failure risk propagation and protection schemes in coupled systems," Chaos, Solitons \& Fractals, vol. 80, pp. 62-75, 2015.

[7] L. Lü, M. Medo, C. H. Yeung, Y.-C. Zhang, Z.-K. Zhang, and T. Zhou, "Recommender systems," Physics Reports, vol. 519, no. 1, pp. 1-49, 2012.

[8] P. Holme and J. Saramäki, “Temporal networks," Physics Reports, vol. 519, no. 3, pp. 97-125, 2012.

[9] S. Tayur, R. Ganeshan, and M. Magazine, Quantitative Models for Supply Chain Management, vol. 17, Springer Science \& Business Media, Berlin, Germany, 2012.

[10] D. J. Watts and S. H. Strogatz, "Collective dynamics of 'smallworld' networks,” Nature, vol. 393, no. 6684, pp. 440-442, 1998.

[11] A.-L. Barabási and R. Albert, "Emergence of scaling in random networks," Science, vol. 286, no. 5439, pp. 509-512, 1999.

[12] Q. Liu and J. Yi, "The research of the social network evolution based on the evolutionary game theory," Acta Physica Sinica, vol. 62, no. 23, Article ID 238902, 2013.
[13] R. R. Wilkinson and K. J. Sharkey, "Message passing and moment closure for susceptible-infected-recovered epidemics on finite networks," Physical Review E, vol. 89, no. 2, Article ID 022808, 6 pages, 2014.

[14] X.-Z. Peng, H. Yao, J. Du, C. Ding, and Z.-H. Zhang, "Study on cascading invulnerability of multi-coupling-links coupled networks based on time-delay coupled map lattices model," Acta Physica Sinica, vol. 63, no. 7, Article ID 078901, 2014.

[15] Y.-R. Song, G.-P. Jiang, and Y.-W. Gong, "Epidemic propagation on adaptive coevolutionary networks with preferential localworld reconnecting strategy," Chinese Physics B, vol. 22, no. 4, Article ID 040205, 2013.

[16] C. Y. Xia, Z. X. Liu, Z. Q. Chen, and Z. Z. Yuan, "SIRS epidemic model with direct immunization on complex networks," Control and Decision, vol. 23, no. 4, pp. 468-472, 2008.

[17] D. H. Zanette, "Dynamics of rumor propagation on small-world networks," Physical Review E, vol. 65, no. 4, Article ID 041908, 2002.

[18] H. J. Shi, Z. S. Duan, and G. R. Chen, "An SIS model with infective medium on complex networks," Physica A: Statistical Mechanics and Its Applications, vol. 387, no. 8-9, pp. 2133-2144, 2008 .

[19] Z. Li, G.-A. Xu, X.-F. Ban, Y. Zhang, and Z.-M. Hu, “Complex information system security risk propagation research based on cellular automata," Acta Physica Sinica, vol. 62, no. 20, Article ID 200203, 2013.

[20] X. Chu, Z. Zhang, J. Guan, and S. Zhou, "Epidemic spreading with nonlinear infectivity in weighted scale-free networks," Physica A: Statistical Mechanics and Its Applications, vol. 390, no. 3, pp. 471-481, 2011.

[21] G. Zhu, X. Fu, and G. Chen, "Global attractivity of a networkbased epidemic SIS model with nonlinear infectivity," Communications in Nonlinear Science and Numerical Simulation, vol. 17, no. 6, pp. 2588-2594, 2012.

[22] Y.-Z. Zhou, Z.-H. Liu, and J. Zhou, "Periodic wave of epidemic spreading in community networks," Chinese Physics Letters, vol. 24, no. 2, p. 581, 2007.

[23] H. Zhang and W. Y. Zhang, "Time-delayed information can induce the periodic outbreaks of infectious diseases," Science China Physics, Mechanics and Astronomy, vol. 42, pp. 631-638, 2012.

[24] P. Holme and B. J. Kim, "Growing scale-free networks with tunable clustering," Physical Review E, vol. 65, no. 2, Article ID 026107, 4 pages, 2002.

[25] K.-I. Goh, B. Kahng, and D. Kim, "Universal behavior of load distribution in scale-free networks," Physical Review Letters, vol. 87, no. 27, Article ID 278701, 4 pages, 2001. 


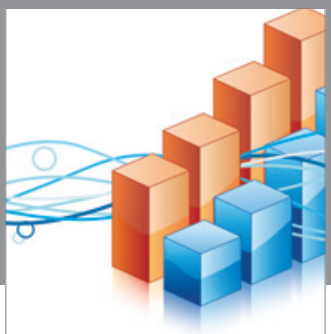

Advances in

Operations Research

vatem alat4

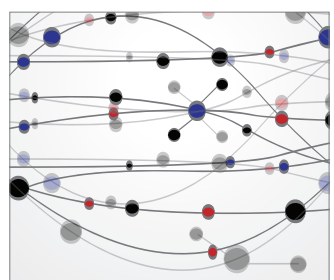

\section{The Scientific} World Journal
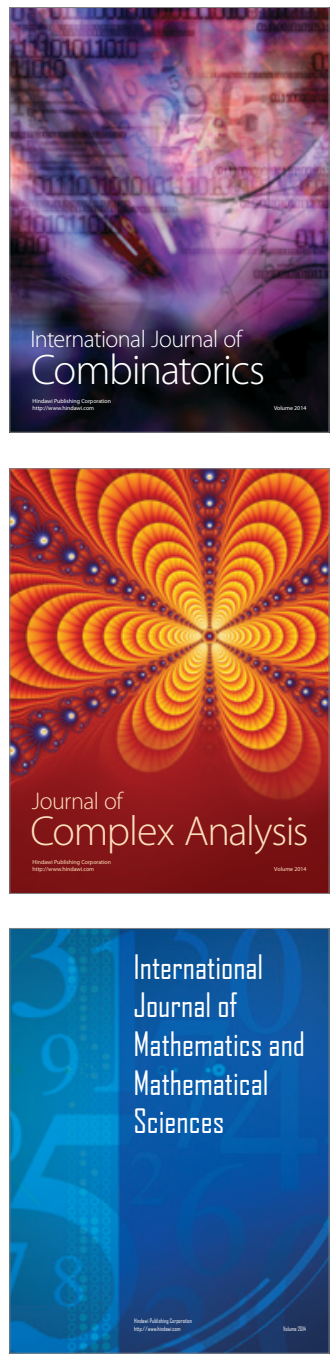
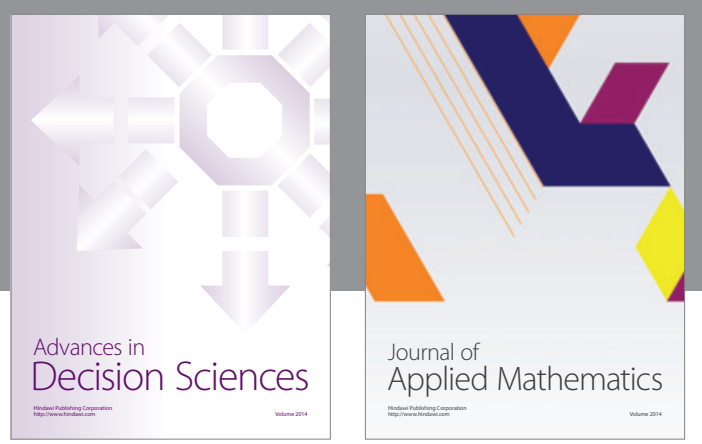

Algebra

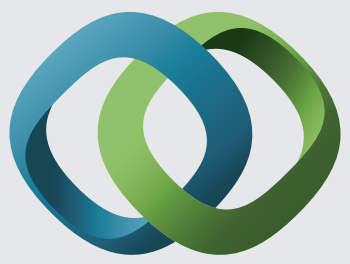

\section{Hindawi}

Submit your manuscripts at

http://www.hindawi.com
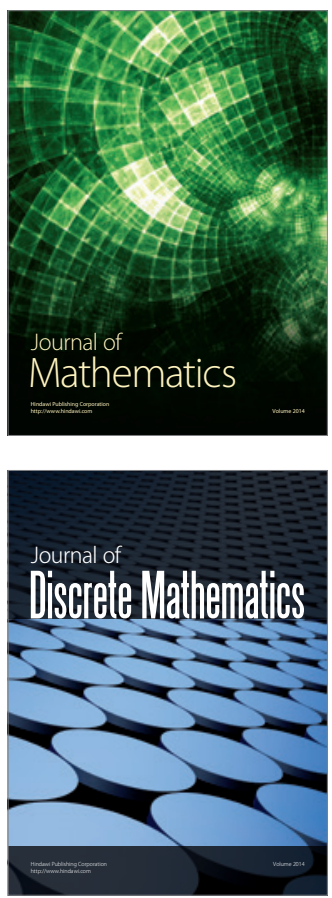

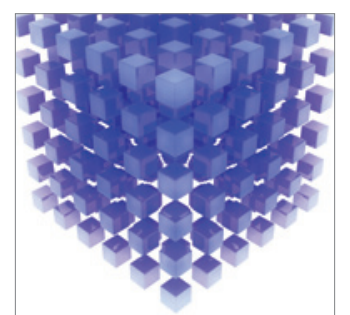

Mathematical Problems in Engineering
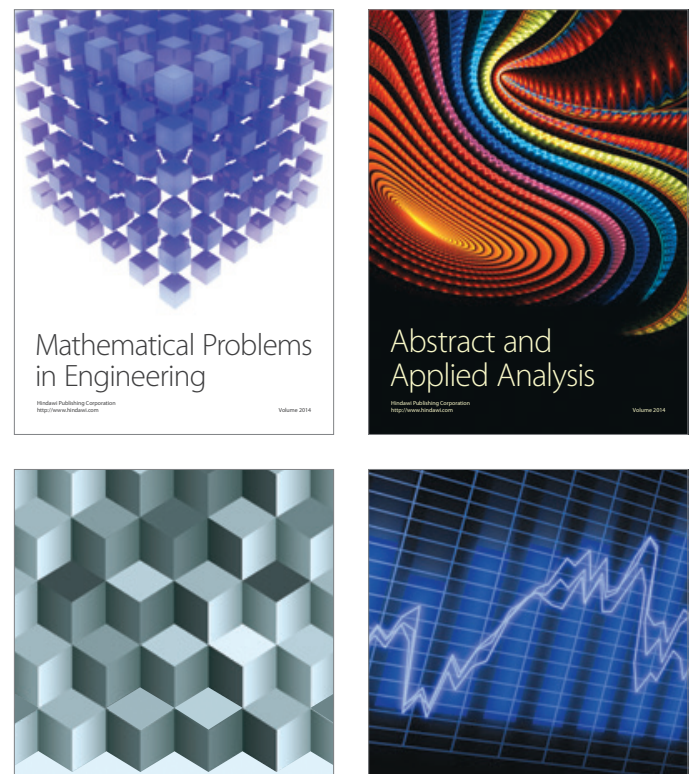

Journal of

Function Spaces

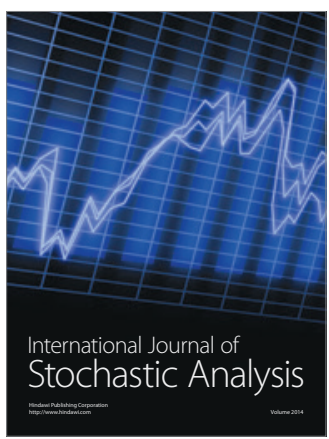

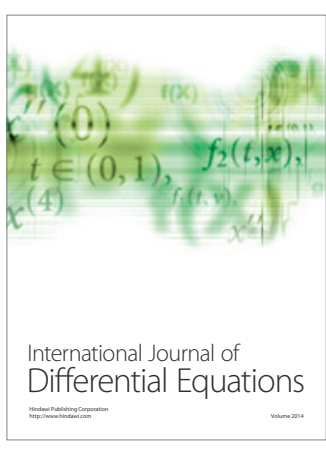
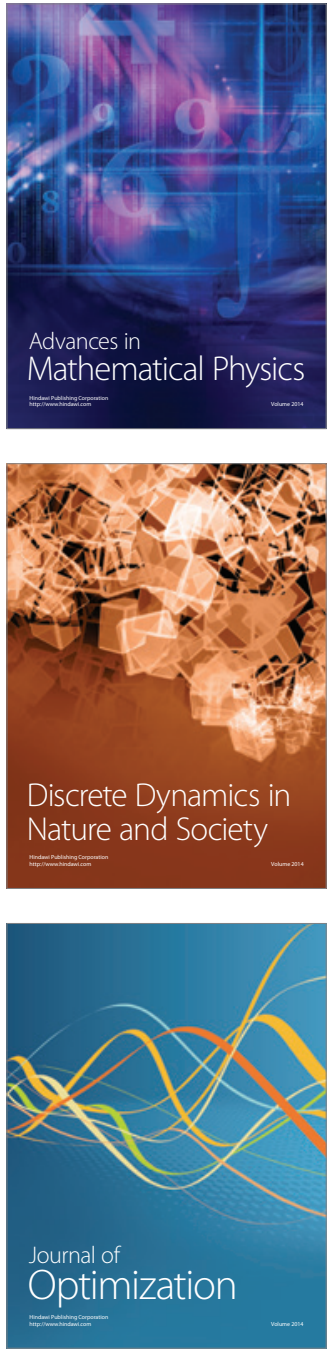